\title{
Graft Reconditioning before Liver Transplantation
}

\author{
Dieter P. Hoyer Thomas Minor \\ General, Visceral and Transplantation Surgery, University Hospital Essen, Essen, Germany
}

\section{Keywords}

Reconditioning - Liver preservation - Extended criteria donors · Liver viability assessment

\section{Summary}

Background: The high demand for livers for transplantation has led to organs of limited quality being accepted to expand the donor pool. This is associated with inferior outcomes due to more pronounced preservation injury. Accordingly, recent research has aimed to develop preservation modalities for improved preservation as well as strategies for liver viability assessment and liver reconditioning. Methods: The PubMed database was searched using the terms 'perfusion', 'liver', 'preservation', and 'reconditioning' in various combinations, and the according literature was reviewed. Results: Several perfusion techniques have been developed in recent years with the potential for liver reconditioning. Preclinical and first emerging clinical data suggest feasibility, safety, and superiority over the current gold standard of cold storage. Conclusion: This review outlines current advances in the field of liver preservation with an emphasis on liver reconditioning methods.

(C) 2016 S. Karger GmbH, Freiburg

\section{Introduction}

Liver transplantation (LT) has evolved from an experimental therapeutic approach in the 20th century to standard therapy nowadays. The extensive knowledge and expertise that has been acquired throughout the last decades is reflected by reports that include hundreds and even thousands of patients in single institutions [1-3]. Such reports demonstrate the continuous and ongoing improvements in LT outcomes. The large amount of patients in need of transplantation was confirmed by the official data report of the United Network for Organ Sharing (UNOS) [4] and Eurotransplant (ET) [5].

Unfortunately, the number of organ donors still does not match the number of organs needed, resulting in long waiting times. Accordingly, not all transplant candidates survive until an organ becomes available, and the mortality on the waiting lists remains high [6-8]. To overcome this situation, several ways to increase the donor pool have been suggested, with the acceptance of livers of limited quality being the predominant way of making additional organs available [9-11]. Subsequently, the risk associated with LT has increased as such organs are more prone to ischemia/reperfusion injury after implantation [12-15]. This can lead to severe or even extreme allograft injury, resulting in increased morbidity and mortality immediately post transplantation [16]. While elaborate methods and scoring systems to achieve a reasonable donor-recipient match in such a context have been developed [17-19], the underlying problem of marginal graft quality calls for a solution.

Especially the period of preservation requires intervention, as during this time the organs are exposed to ischemia which is a key trigger of reperfusion injury. Accordingly, the focus of recent and ongoing research has been the improvement of preservation. This manuscript reviews current advances in the field of liver preservation with an emphasis on liver reconditioning methods.

Data for this review were collected by searching the PubMed database using the search terms 'perfusion', 'liver', 'preservation', and 'reconditioning' in various combinations. Relevant cross references not previously found were contemplated as well. Included in this review were relevant articles written in English. Not all studies presenting similar or identical messages may be cited here.

\section{Static Preservation}

\section{Cold Storage}

For decades, the gold standard of organ preservation in solid organ transplantation has been cold storage (CS) in which an

\section{KARGER}

(C) 2016 S. Karger GmbH, Freiburg

Fax +497614520714 
organ is flushed with a precooled solution and stored statically submerged in this solution on ice [20]. The cooling of organs to $4{ }^{\circ} \mathrm{C}$ results in a reduction in metabolic activity to approximately $5 \%$ and subsequent protection from gross ischemic injury. So far, it is not possible to completely inactivate the metabolism so that energy substrates like adenosine triphosphate (ATP) linearly decrease over time together with hepatocyte viability [20], resulting in a risk of organ dysfunction after LT [21]. Additionally, the low temperature itself mitigates pathophysiological alterations leading to cold-induced organ injury, which is most detrimental in organs of marginal quality.

While superiority of one preservation solution over others is still a subject of debate [22-24], the development of different compositions of perfusion solution over the years [25-30] has made this simple method efficacious and allowed LT to become a lifesaving procedure around the world. The approximation of the temperature to the freezing point of liver tissue at -0.8 degrees [31] and the supercooling of the allografts in an unfrozen state by generation of a surrounding electrostatic field [32] are recent developments for the further improvement of CS.

\section{Dynamic Preservation}

The idea to cannulate and perfuse organs for preservation purposes is not new. Indeed, first investigations of ex vivo organ perfusion were carried out at the beginning of the transplantation age [33-36], and the first successful LT by Starzl et al. [37] utilized hypothermic machine perfusion for the preservation of the allografts. While these methods were not used for years due to the much simpler and cost-effective successful application of CS, a renewed interest was aroused in the early 1990s in search of optimized preservation given the increased use of marginal liver allografts.

Of particular interest is the concept of endischemic organ reconditioning at the end of the CS period immediately before transplantation. Such an approach utilizes existing logistic structures and does not require training of specialized staff to oversee elaborate perfusion techniques during transportation. Furthermore, interventions on the allograft are performed at the accepting transplant center only, with optimal conditions to treat potential complications. Unfortunately, not all dynamic techniques are equally suitable for such an application.

\section{Hypothermic Machine Perfusion}

Hypothermic machine perfusion (HMP) is carried out at temperatures similar to static CS, therefore considerably reducing the metabolic demands of the liver allograft. Optimal conditions and modalities are still a subject of debate. Perfusion pressures need to be reduced to sub-physiological levels to avoid injury of stiff vessels and tissues in the cold environment. Experimental investigations suggested optimal results with $25 \%$ of physiological pressure with regard to the proportion of complete perfusion without any en- dothelial damages [38]. This was confirmed by simulation studies which additionally showed that the viscosity of the perfusion solutions in this setting has minor effects on the shear stress in the vessels during HMP [39]. While the majority of experiments in HMP utilized original or modified UW (University of Wisconsin) solution or UW machine perfusion solution, other studies used IGL (Institut George Lopez), Celsior, or HTK (histidine-tryptophanketoglutarate) solution. Indeed, an ideal perfusate for liver HMP has yet to be determined [40]. Current research is showing promising results for the feasibility and efficacy of new solutions like polysol [41], vasosol [42], or HTK-N [43, 44] for HMP. Further interesting and potentially important aspects like the flow competition between the portal vein and the hepatic artery during HMP [45] also need further clarification to determine whether preference should be given to single perfusion of the portal vein or dual perfusion of the portal vein and the hepatic artery in HMP.

Lastly, endischemic HMP was carried out in discarded human livers and compared to CS, reporting specific mRNA profiles and reduced aspartate transaminase (AST) and lactate dehydrogenase (LDH) release during warm ex-vivo reperfusion. No morphological differences were found [46]. In discarded steatotic grafts coldstored for $13 \mathrm{~h}$ before initiation of HMP, an increased release of AST and LDH into the perfusate but similar vascular resistances and metabolic profiles in comparison with transplantable human livers were noted [47].

Predominantly, two groups have pushed the envelope in HMP in recent years and advanced progress to clinical application. The approach of Guarrera et al. [48] utilizes an HMP device with dual continuous centrifugal perfusion of the portal vein and hepatic artery with vasosol. Perfusion is carried out in a flow-controlled manner without active oxygenation. Graft procurement and transportation to the transplanting center are performed in a standard fashion, resulting in an endischemic application of HMP.

The first clinical application in 20 recipients reported in 2010 demonstrated rates of early allograft dysfunction (EAD) [49] of 5\% in the HMP group versus $25 \%$ in controls $(p=0.08)$ and significantly lower serum injury markers in the HMP group [48]. These preliminary data were further confirmed by the extension of the application to another 31 marginal liver allografts: Here, rates of EAD were $19 \%$ in the HMP group versus $30 \%$ in the control group $(\mathrm{p}=0.384)$. Interestingly, a post hoc analysis revealed significantly less biliary complications in the HMP group versus the CS group $(\mathrm{p}=0.016)$. The authors concluded that HMP provided safe and reliable preservation in marginal liver allografts [50]. In a review by the same group, it was suggested that HMP's advantages are not limited to maintaining a patent microvasculature, but it also provides a tool for extracorporeal treatment, assessment, and clearance of waste products. In spite of the fact that no conclusive data on all these aspects is available as yet, the authors expect HMP to become the next gold standard for liver preservation [13, 51].

In contrast to Guarrera et al. [48], the group of Dutkowski et al. [52-56] utilizes pressure-controlled single perfusion via the portal vein with oxygenated modified UW solution for endischemic hypothermic oxygenated perfusion (HOPE). In a series of publica- 
tions, a successful profile for HMP as a reconditioning tool has been shown by the group with a focus on liver allografts from DCD (donation after cardiac death) donors. In rodent DCD models, reperfusion injury was significantly decreased by HOPE treatment according to hepatocyte injury, Kupffer cell activation, and endothelial cell activation [52]. The protective mechanisms of HOPE were analyzed in pig models where oxygenation under hypothermic conditions protects from mitochondrial and nuclear injury by downregulation of mitochondrial activity before reperfusion. Furthermore, cold perfusion itself prevented endothelial damage under low pressure conditions, independently of additional oxygenation [53]. In addition to that, an elegant rodent LT model by the same group demonstrated that application of HOPE blunted the alloimmune response in the recipient, extending the beneficial effects from rescuing marginal grafts to preventing rejection [54].

First clinical applications of HOPE in the DCD setting have been accomplished: Leakage of liver enzymes, kidney function, and length of intensive care unit and hospital stay were observed to be comparable or better than with matched liver grafts from DBD (donation after brain death) donors. It is of special interest that no evidence of biliary complications occurred during follow-up in this DCD setting [55]. These data were confirmed by subsequent clinical investigations which again showed positive outcomes in terms of graft function and biliary complications [56]. Accordingly, a randomized controlled multicenter trial has been initiated based on these results and is currently recruiting patients (NCT01317342).

Contrary to these positive data, other groups reported that oxygenated HMP causes endothelial and Kupffer cell injury that may ultimately lead to the failure of such grafts in DCD pig models [57].

Several markers, which can be acquired during HMP, have been suggested to correlate with outcome after LT, so that viability testing of the allograft seems possible before LT. Potential markers investigated so far include levels of aspartate aminotransferase, alanine, histidine, acidosis of the perfusate, liver fatty acid-binding protein, redox-active iron, and arterial vascular resistance $[58,59]$.

\section{Subnormothermic Machine Perfusion}

In the subnormothermic temperature range, the metabolism of an isolated organ is not as depressed as under hypothermic conditions. Accordingly, demands on preservation modalities are higher, and e.g. sufficient oxygenation is a prerequisite for successful application. Theoretically, these temperatures reduce the cold-induced organ injury and enable more detailed organ assessments and interventions due to the semi-active metabolic state. At the same time, this modality of preservation is far less demanding than preservation at physiological or near-physiological temperatures and can usually be carried out with cell-free preservation solutions. In accordance with these considerations, no signs of anaerobiosis (HIF-1 $\alpha$ ) or liver injury were observed during oxygenated perfusion at 10 or $20^{\circ} \mathrm{C}$ in contrast to perfusion at 30 or $37^{\circ} \mathrm{C}[60]$. Other rodent models demonstrated superior results at $21^{\circ} \mathrm{C}$ compared to lower temperatures in DBD $[61,62]$ and DCD [63] mod- els. This indicates an important shift in hepatic metabolism between 20 and $30^{\circ} \mathrm{C}$, explaining the favorable ATP concentrations after subnormothermic machine perfusion (SNTM).

While demonstrating promising results, the basic conditions of SNTM are still a subject of debate. Similarly to HMP, the optimal preservation solution for SNTM has not yet been defined. Continuous perfusion throughout the entire preservation period successfully reduces the rate of apoptosis in the sinusoids of steatotic livers, resulting in favorable perfusion dynamics [12]. In addition, continuous SNTM demonstrated recovery of ischemically damaged rat livers, with low aminotransferases after reperfusion suggesting beneficial effects. However, in this model, rising bilirubin levels suggested inadequate prevention of ischemia or hypothermia-induced biliary damage [64]. Application in pig models demonstrated significantly reduced aminotransferases, LDH, and lactate during reperfusion [65].

Indeed, the endischemic application of SNTM in rodent models was sufficient to recover ATP homeostasis, while the length of the preceding period of CS correlated with vascular resistance during SNTM and the maximum level of ATP recovery. Survival of rats increased significantly from 50 to $100 \%$ by application of reconditioning SNTM when a CS period of $72 \mathrm{~h}$ was not exceeded, implying a limit to the reconditioning potential of SNTM [66].

In a different model with subnormothermic temperatures of up to $33{ }^{\circ} \mathrm{C}$ in pigs, the favorable effects of endischemic SNTM were observed clearly in DBD donors with significantly lower AST and bilirubin peaks and endothelial cell and bile duct injury even in grafts with minimal CS time [67]. In DCD donors, the beneficial effects of reconditioning SNTM were less pronounced but still pointed to an improved preservation of biliary structures [68].

The technical details of endischemic SNTM have recently been thoroughly documented $[69,70]$ and further investigated in 7 discarded human livers: biochemical and microscopic assessment demonstrated minimal injury sustained during perfusion. On the other hand, improved oxygen uptake, lactate levels, and ATP content were observed, and actual liver function during SNTM was reflected by urea, albumin, and bile production. The authors concluded that SNTM maintains liver function with minimal injury and sustains or improves liver function during reperfusion [71].

\section{Normothermic Machine Perfusion}

Normothermic machine perfusion (NMP) has been suggested to allow optimized viability assessment of the isolated graft and prevent any cold-induced organ injury. However, successful NMP requires an elaborated perfusion system meeting the demands of a metabolically fully active organ. Therefore, oxygenized blood containing several adjuncts is usually used as perfusion solution. Recent published data showed feasibility and beneficial effects in terms of bile duct injury and arterial perfusion in DCD liver grafts with acellular perfusion solution [72] in contrast to former studies which indicated that acellular oxygenized solutions fail to meet the demands of organs at 30 or $37^{\circ} \mathrm{C}[60]$. Differences in solutions and 
perfusion models would contribute to this interesting aspect which needs further clarification in the future.

Early efforts to carry out NMP aimed at the evaluation of hepatic function $[73,74]$ under normal metabolic conditions and showed that mammalian livers could be kept completely functional outside the body for more than $4 \mathrm{~h}$ [75]. Subsequently, longer perfusion periods were tested and demonstrated successful transplantation of both normal livers and those with substantial ischemic damage [76]. Recent data in DCD models confirmed the results and showed that NMP improves metabolic and functional parameters of livers with short or extended warm ischemia times compared with livers subjected to comparable cold ischemia times [77]. Parameters indicative of liver injury such as aminotransferase elevation and reduced ATP concentrations after warm ischemic injury are stabilized or even recovered after initiation of NMP, reaching comparable levels to controls after $1 \mathrm{~h}$ of NMP. These data were in agreement with histology and gross morphology which confirmed recovery of the ischemic grafts [78]. In pig livers [79], evidence of function during NMP was demonstrated in terms of bile production, paracetamol removal, and maintenance of normal ammonia and lactate levels. Staining for Ki-67 in extrahepatic bile ducts showed biliary epithelial regeneration as an endpoint of the beneficial effects of NMP in DCD livers, suggesting that the development of biliary complications might therefore be reduced [80]. Moreover, extension of NMP to steatotic organs demonstrated successful preservation for prolonged periods and facilitation of a reduction in hepatic steatosis [81].

Several preclinical studies investigated possible markers during perfusion for the assessment of liver viability before LT, which included urea, lactate, glucose, ornithine, arginine, albumin, and tyrosine [82].

Investigations with discarded human grafts showed that NMP is technically feasible and presumably allows assessment of graft viability, opening new avenues for organ selection, interventions, and conditioning before transplantation $[83,84]$.

However, the high technical demands of NMP [85] have to be kept in mind, and former published studies reporting beneficial effects in part failed to provide the elementary perfusion prerequisites for harmless application $[86,87]$.

Furthermore, the distinct mechanisms of protection during NMP are not yet completely understood. Current studies investigating the major pathways of energy metabolism are trying to overcome these shortcomings and have concluded that $2 \mathrm{~h}$ of NMP is adequate to exploit the potential of energetic recovery in DCD liver allografts [88].

The results of first applications of NMP in clinical LT have been published recently [89]. The data indicated a significantly lower median AST peak in the first 7 days after LT in the NMP group when compared with a matched control cohort. Based on these data, the authors stressed the safety and feasibility of this technology from retrieval to transplantation, including transportation [90].

So far, NMP has been primarily utilized as a continuous preservation method with challenging demands on logistics and staff. Unfortunately, little data focuses on NMP as an endischemic recondi- tioning tool. While in a rodent model of endischemic NMP tissue ATP levels were replete and detrimental effects were prevented [91], such data were not confirmed in a porcine DCD model which demonstrated failure of endischemic NMP to resuscitate porcine livers after $60 \mathrm{~min}$ of warm ischemia and $4 \mathrm{~h}$ of cold preservation. In this study, the authors concluded that even a short period of cold ischemia is significantly deleterious to the function of ischemically damaged livers [92]. The consideration that any form of endischemic NMP represents an early graft reperfusion, especially when executed with blood, might explain this matter. Therefore, current data indicates that NMP has limited potential as a reconditioning preservation method, and is rather more suitable for continuous conditioning throughout the entire preservation period.

\section{Controlled Oxygenated Rewarming}

Experimental evidence suggests an important role for the abrupt temperature shift from hypothermia to normothermia incurred on reperfusion of liver grafts in triggering mitochondrial dysfunction [93]. This temperature shift has been delineated as a genuine factor contributing to reperfusion injury and graft dysfunction after LT. The extent of this rewarming injury seems to be dependent on the duration and degree of the preceding hypothermia. Interestingly, rewarming injury was not observed in isolated hepatocytes after storage above $16^{\circ} \mathrm{C}$ [94] which therefore seems to be an important threshold for all cold and subnormothermic preservation modalities. Recent studies in pig livers have shown that slowly increasing the temperature using controlled oxygenated rewarming (COR) - and thereby slowly increasing metabolic activity - effectively improves graft function upon reperfusion [44]. The transient phase of cold to mid-thermic perfusion seems to allow for a gentle restitution of mitochondrial function at a limited workload, promoting superior restitution of hepatocellular function. The trigger for mitochondrial dysfunction upon normothermic reperfusion seems reduced.

Other study groups demonstrated similar beneficial effects of COR [95], again in porcine livers, where AST, LDH, and hyaluronic acid levels were significantly lower after reperfusion.

Performing COR at temperatures of up to $21^{\circ} \mathrm{C}$ is based on the aforementioned metabolic shift of liver allografts between 20 and $30{ }^{\circ} \mathrm{C}$ with favorable outcomes at the $20^{\circ} \mathrm{C}$ level [60]. Additionally, the important threshold for rewarming injuries of $16^{\circ} \mathrm{C}$ [94] is clearly crossed.

The results of the first clinical application of COR have recently been published [96]. In accordance with the experimental investigations, a significant reduction in AST peaks after transplantation, lower rates of EAD, and better survival after 6 months were observed in the COR group compared to age-matched controls. Additionally, correlating perfusion parameters with outcome suggested an expedient liver viability assessment during endischemic perfusion.

COR is a strictly endischemic reconditioning method, as cooler preceding temperatures are required for a reasonable application. 
Therefore, the advantages of reconditioning protocols completely apply to this method.

\section{Comparison of Current Reconditioning and Preservation Strategies}

Extensive research has been carried out to develop the aforementioned preservation modalities. First comparative studies are currently emerging and will give further clarification as to optimal reconditioning and preservation strategies.

HOPE as an endischemic reconditioning method was recently compared to continuous NMP in a clinically relevant DCD rodent model. While both methods showed superior results in comparison with CS, HOPE demonstrated superiority over NMP in terms of graft survival through protection from hepatocyte and non-parenchymal cell injury and therefore a modulation of the innate immunity and endothelial cell activation [97].

Already the first publication on COR in porcine livers compared this method to other preservation methods, including CS, endischemic HMP, and endischemic SNTM, demonstrating best organ preservation after COR [44]. This is supported by data dem- onstrating lower AST levels in perfusates of COR compared to continuous or endischemic HMP and lower values of AST and LDH coinciding with less histologically proven hepatocellular necrosis $2 \mathrm{~h}$ after reperfusion in a DCD porcine model [98]. Further comparisons of COR with NMP were carried out by our group in a porcine ex-vivo reperfusion model (unpublished data): Better mitochondrial integrity resulted in better energetic recovery, less hepatocellular injury, and ultimately superior function after COR.

Ultimately, the combination of different preservation methods might provide the best preservation conditions. However, little data is available so far, and technical and logistic challenges need to be overcome for such concepts to thrive. In a first publication, Banan et al. [99] suggested that if livers have to undergo a period of cold preservation, a combination of COR and NMP may greatly reduce damage associated with reperfusion by minimizing hepatocellular damage, Kupffer cell activation, and sinusoidal endothelial cell dysfunction.

\section{Disclosure Statement}

The authors have no conflict of interest to disclose.

\section{References}

1 Salizzoni M, Cerutti E, Romagnoli R, et al: The first one thousand liver transplants in Turin: a single-center experience in Italy. Transpl Int 2005;18:1328-1335.

$\checkmark 2$ Uemura T, Randall HB, Sanchez EQ, et al: Liver retransplantation for primary nonfunction: analysis of a 20-year single-center experience. Liver Transpl 2007; $13: 227-233$

3 Agopian VG, Petrowsky H, Kaldas FM, et al: The evolution of liver transplantation during 3 decades: analysis of 5,347 consecutive liver transplants at a single center. Ann Surg 2013;258:409-421.

4 www.unos.org/data/2016/

5 www.eurotransplant.org/cms/index.php?page = yearlystats; 2016

6 Sharma P, Schaubel DE, Gong Q, et al: End-stage liver disease candidates at the highest model for end-stage liver disease scores have higher wait-list mortality than status-1A candidates. Hepatology 2012;55:192-198.

7 Kim WR, Biggins SW, Kremers WK, et al: Hyponatremia and mortality among patients on the livertransplant waiting list. N Engl J Med 2008;359:10181026.

8 Kwong AJ, Lai JC, Dodge JL, Roberts JP: Outcomes for liver transplant candidates listed with low model for end-stage liver disease score. Liver Transpl 2015;21: 1403-1409.

7 Sotiropoulos GC, Paul A, Molmenti E, et al: Liver transplantation for hepatocellular carcinoma in cirrhosis within the Eurotransplant area: an additional option with 'livers that nobody wants'. Transplantation 2005;80:897-902.

10 Hoyer DP, Paul A, Saner F, et al: Safely expanding the donor pool: brain dead donors with history of temporary cardiac arrest. Liver Int 2015;35:1756-1763.
11 Radunz S, Paul A, Nowak K, et al: Liver transplantation using donor organs with markedly elevated liver enzymes: how far can we go? Liver Int 2011;31:10211027.

12 Boncompagni E, Gini E, Ferrigno A, et al: Decreased apoptosis in fatty livers submitted to subnormothermic machine-perfusion respect to cold storage. Eur J Histochem 2011;55:e40.

13 Graham JA, Guarrera JV: 'Resuscitation' of marginal liver allografts for transplantation with machine perfusion technology. J Hepatol 2014;61:418-431.

14 Busuttil RW, Tanaka K: The utility of marginal donors in liver transplantation. Liver Transplant 2003;9:651663.

15 Ravikumar R, Leuvenink H, Friend PJ: Normothermic liver preservation: a new paradigm? Transpl Int 2015; 28:690-699.

16 Hoyer DP, Sotiropoulos GC, Saner FH, et al: MELD at POD 1 as a predictor of outcome in liver allografts with peak AST >5,000 U/l. Transpl Int 2014;27:12851293.

17 Hoyer DP, Paul A, Gallinat A, et al: Donor information based prediction of early allograft dysfunction and outcome in liver transplantation. Liver Int 2015;35: $156-163$.

18 Briceño J, Ciria R, de la Mata M: Donor-recipient matching: myths and realities. J Hepatol 2013;58:811820.

19 Dutkowski P, Oberkofler CE, Slankamenac K, et al: Are there better guidelines for allocation in liver transplantation? A novel score targeting justice and utility in the model for end-stage liver disease era. Ann Surg 2011;254:745-753.

20 Berendsen TA, Izamis ML, Xu H, et al: Hepatocyte viability and adenosine triphosphate content decrease linearly over time during conventional cold storage of rat liver grafts. Transplant Proc 2011;43:1484-1488.
21 Perera MTPR, Richards DA, Silva MA, et al: Comparison of energy metabolism in liver grafts from donors after circulatory death and donors after brain death during cold storage and reperfusion. Br J Surg 2014; 101:775.

22 Adam R, Delvart V, Karam V, et al: Compared efficacy of preservation solutions in liver transplantation: a long-term graft outcome study from the European Liver Transplant Registry. Am J Transplant 2015;15: 395-406.

23 O'Callaghan JM, Morgan RD, Knight SR, Morris PJ The effect of preservation solutions for storage of liver allografts on transplant outcomes: a systematic review and meta-analysis. Ann Surg 2014;260:46-55.

24 Latchana N, Peck JR, Whitson BA, et al: Preservation solutions used during abdominal transplantation: current status and outcomes. World J Transplant 2015;5: 154-164.

25 Zaouali MA, Ben Abdennebi H, Padrissa-Altés S, et al: Pharmacological strategies against cold ischemia reperfusion injury. Expert Opin Pharmacother 2010;11: 537-555.

26 Lee CY, Mangino MJ: Preservation methods for kidney and liver. Organogenesis 2009;5:105-112.

27 Olthoff KM, Millis JM, Imagawa DK, et al: Comparison of UW solution and Euro-Collins solutions for cold preservation of human liver grafts. Transplantation 1990;49:284-290.

28 D'Alessandro AM, Kalayoglu M, Sollinger HW, et al: Current status of organ preservation with University of Wisconsin solution. Arch Pathol Lab Med 1991;115: 306-310.

29 Ben Abdennebi H, Elrassi Z, Scoazec J-Y, et al: Evaluation of IGL-1 preservation solution using an orthotopic liver transplantation model. World J Gastroenterol 2006;12:5326-5330. 
-30 Tabka D, Bejaoui M, Javellaud J, et al: Effects of Institut Georges Lopez-1 and Celsior preservation solutions on liver graft injury. World J Gastroenterol 2015;21: 4159-4168.

- 31 Yoshida K, Matsui Y, Wei T, et al: A novel conception for liver preservation at a temperature just above freezing point. J Surg Res 1999;81:216-223.

32 Monzen K, Hosoda T, Hayashi D, et al: The use of a supercooling refrigerator improves the preservation of organ grafts. Biochem Biophys Res Commun 2005; 337:534-539.

33 Slapak M, Wigmore RA, MacLean LD: Twenty-four hour liver preservation by the use of continuous pulsatile perfusion and hyperbaric oxygen. Transplantation 1967;5(suppl):1154-1158.

-34 Lindbergh CA, Perry VP, Malinin TI, Mouer GH: An apparatus for the pulsating perfusion of whole organs. Cryobiology 1966;3:252-260.

35 Belzer FO, May R, Berry MN, Lee JC: Short term preservation of porcine livers. J Surg Res 1970;10:55-61.

-36 Belzer FO, Ashby BS, Gulyassy PF, Powell M: Successful seventeen-hour preservation and transplantation of human-cadaver kidney. N Engl J Med 1968;278:608-610.

-37 Starzl TE, Groth CG, Brettschneider L, et al: Extended survival in 3 cases of orthotopic homotransplantation of the human liver. Surgery 1968;63:549-563.

38 't Hart NA, der van Plaats A, Leuvenink HGD, et al: Determination of an adequate perfusion pressure for continuous dual vessel hypothermic machine perfusion of the rat liver. Transpl Int 2007;20:343-352.

39 Debbaut C, Monbaliu D, Casteleyn C, et al: From vascular corrosion cast to electrical analog model for the study of human liver hemodynamics and perfusion. IEEE Trans Biomed Eng 2011;58:25-35.

40 Schlegel A, Kron P, Dutkowski P: Hypothermic oxygenated liver perfusion: basic mechanisms and clinical application. Curr Tansplant Rep 2015;2:52-62.

41 Bessems M, Doorschodt BM, van Marle J, et al: Improved machine perfusion preservation of the nonheart-beating donor rat liver using Polysol: a new machine perfusion preservation solution. Liver Transpl 2005;11:1379-1388.

42 Bae C, Pichardo EM, Huang H, et al: The benefits of hypothermic machine perfusion are enhanced with Vasosol and $\alpha$-tocopherol in rodent donation after cardiac death livers. Transplant Proc 2014;46:1560-1566.

43 Fingas CD, Wu S, Gu Y, et al: Assessment of a chloride-poor versus a chloride-containing version of a modified histidine-tryptophan-ketoglutarate solution in a rat liver transplantation model. Liver Transpl 2011; 17:650-660.

44 Minor T, Efferz P, Fox M, et al: Controlled oxygenated rewarming of cold stored liver grafts by thermally graduated machine perfusion prior to reperfusion. Am J Transplant 2013;13:1450-1460.

45 Monbaliu DR, Debbaut C, Hillewaert WJ, et al: Flow competition between hepatic arterial and portal venous flow during hypothermic machine perfusion preservation of porcine livers. Int J Artif Organs 2012; 35:119-131.

46 Vekemans K, van Pelt J, Komuta M, et al: Attempt to rescue discarded human liver grafts by end ischemic hypothermic oxygenated machine perfusion. Trans plant Proc 2011;43:3455-3459.

47 Monbaliu D, Liu Q, Libbrecht L, et al: Preserving the morphology and evaluating the quality of liver grafts by hypothermic machine perfusion: a proof-of-concept study using discarded human livers. Liver Transpl 2012;18:1495-1507.

48 Guarrera JV, Henry SD, Samstein B, et al: Hypothermic machine preservation in human liver transplantation: the first clinical series. Am J Transplant 2010;10: 372-381.
49 Olthoff KM, Kulik L, Samstein B, et al: Validation of a current definition of early allograft dysfunction in liver transplant recipients and analysis of risk factors. Liver Transpl 2010;16:943-949.

50 Guarrera JV, Henry SD, Samstein B, et al: Hypothermic machine preservation facilitates successful transplantation of 'orphan' extended criteria donor livers. Am J Transplant 2015;15:161-169.

51 Bae C, Henry SD, Guarrera JV: Is extracorporeal hypothermic machine perfusion of the liver better than the 'good old icebox'? Curr Opin Organ Transplant 2012; 17:137-142.

52 Schlegel A, Graf R, Clavien P-A, Dutkowski P: Hypothermic oxygenated perfusion (HOPE) protects from biliary injury in a rodent model of DCD liver transplantation. J Hepatol 2013;59:984-991.

53 Schlegel A, de Rougemont O, Graf R, et al: Protective mechanisms of end-ischemic cold machine perfusion in DCD liver grafts. J Hepatol 2013;58:278-286.

54 Schlegel A, Kron P, Graf R, et al: Hypothermic Oxygenated Perfusion (HOPE) downregulates the immune response in a rat model of liver transplantation. Ann Surg 2014;260:931-937.

55 Dutkowski P, Schlegel A, de Oliveira M, et al: HOPE for human liver grafts obtained from donors after cardiac death. J Hepatol 2014;60:765-772.

56 Dutkowski P, Polak WG, Muiesan P, et al: First com parison of hypothermic oxygenated perfusion versus static cold storage of human donation after cardiac death liver transplants: an international-matched case analysis. Ann Surg 2015;262:764-770.

57 Fondevila C, Hessheimer AJ, Maathuis M-HJ, et al: Hypothermic oxygenated machine perfusion in porcine donation after circulatory determination of death liver transplant. Transplantation 2012;94:22-29.

58 Liu Q, Vekemans K, van Pelt J, et al: Discriminate liver warm ischemic injury during hypothermic machine perfusion by proton magnetic resonance spectroscopy: a study in a porcine model. Transplant Proc 2009;41: 3383-3386.

59 Liu Q, Vekemans K, Iania L, et al: Assessing warm ischemic injury of pig livers at hypothermic machine perfusion. J Surg Res 2014;186:379-389.

60 Ferrigno A, Di Pasqua LG, Bianchi A, et al: Metabolic shift in liver: correlation between perfusion temperature and hypoxia inducible factor-1 $\alpha$. World J Gastroenterol 2015;21:1108-1116.

61 Olschewski P, Gass P, Ariyakhagorn V, et al: The influence of storage temperature during machine perfusion on preservation quality of marginal donor livers. Cryobiology 2010;60:337-343.

62 Vairetti M, Ferrigno A, Rizzo V, et al: Subnormothermic machine perfusion protects against rat liver preservation injury: a comparative evaluation with conventional cold storage. Transplant Proc 2007;39:1765-1767.

63 Ferrigno A, Rizzo V, Boncompagni E, et al: Machine perfusion at $20^{\circ} \mathrm{C}$ reduces preservation damage to livers from non-heart beating donors. Cryobiology 2011; 62:152-158.

64 Tolboom H, Izamis M-L, Sharma N, et al: Subnormothermic machine perfusion at both $20^{\circ} \mathrm{C}$ and $30^{\circ} \mathrm{C}$ recovers ischemic rat livers for successful transplantation. J Surg Res 2012;175:149-156.

65 Gringeri E, Bonsignore P, Bassi D, et al: Subnormothermic machine perfusion for non-heart-beating donor liver grafts preservation in a Swine model: a new strategy to increase the donor pool? Transplant Proc 2012;44:2026-2028.

66 Bruinsma BG, Berendsen TA, Izamis M-L, et al: Determination and extension of the limits to static cold storage using subnormothermic machine perfusion. Int J Artif Organs 2013;36:775-780.
7 Spetzler VN, Goldaracena N, Echiverri J, et al: Subnormothermic ex vivo liver perfusion is a safe alternative to cold static storage for preserving standard criteria grafts. Liver Transpl 2016;22:111-119.

68 Knaak JM, Spetzler VN, Goldaracena N, et al: Subnormothermic ex vivo liver perfusion reduces endothelial cell and bile duct injury after donation after cardiac death pig liver transplantation. Liver Transpl 2014;20: 1296-1305.

69 Knaak JM, Spetzler VN, Goldaracena N, et al: Technique of subnormothermic ex vivo liver perfusion for the storage, assessment, and repair of marginal liver grafts. J Vis Exp 2014;(90):e51419.

70 Bruinsma BG, Avruch JH, Weeder PD, et al: Functional human liver preservation and recovery by means of subnormothermic machine perfusion. J Vis Exp 2015; (98).

71 Bruinsma BG, Yeh H, Ozer S, et al: Subnormothermic machine perfusion for ex vivo preservation and recovery of the human liver for transplantation. Am J Transplant 2014;14:1400-1409.

72 Boehnert MU, Yeung JC, Bazerbachi F, et al: Normothermic acellular ex vivo liver perfusion reduces liver and bile duct injury of pig livers retrieved after cardiac death. Am J Transplant 2013;13:1441-1449.

73 Kollmannsberger A, Wahba A, Pfeiffer U, et al: Evaluation of liver function in an improved model of isolated liver perfusion. Life Support Syst 1985;3(suppl 1): 326-330.

74 Bell R, Shiel AG, Dolan P, et al: The evaluation of the isolated perfused liver as a model for the assessment of liver preservation. Aust N Z J Surg 1993;63:44-52.

75 Schön MR, Kollmar O, Wolf S, et al: Liver transplantation after organ preservation with normothermic extracorporeal perfusion. Ann Surg 2001;233:114-123.

76 Brockmann J, Reddy S, Coussios C, et al: Normothermic perfusion: a new paradigm for organ preservation. Ann Surg 2009;250:1-6.

77 Banan B, Chung H, Xiao Z, et al: Normothermic extracorporeal liver perfusion for donation after cardiac death (DCD) livers. Surgery 2015;158:1642-1650.

$78 \mathrm{Xu} \mathrm{H}$, Berendsen T, Kim K, et al: Excorporeal normothermic machine perfusion resuscitates pig DCD livers with extended warm ischemia. J Surg Res 2012;173: e83-88.

79 Bellomo R, Suzuki S, Marino B, et al: Normothermic extracorporeal perfusion of isolated porcine liver after warm ischaemia: a preliminary report. Crit Care Resusc 2012;14:173-176.

80 Liu Q, Nassar A, Farias K, et al: Sanguineous normothermic machine perfusion improves hemodynamics and biliary epithelial regeneration in donation after cardiac death porcine livers. Liver Transpl 2014;20: 987-999.

81 Jamieson RW, Zilvetti M, Roy D, et al: Hepatic steatosis and normothermic perfusion-preliminary experiments in a porcine model. Transplantation 2011;92: 289-295.

82 Perk S, Izamis M-L, Tolboom H, et al: A metabolic index of ischemic injury for perfusion-recovery of cadaveric rat livers. PLoS One 2011;6:e28518.

83 Bellomo R, Marino B, Starkey G, et al: Extended normothermic extracorporeal perfusion of isolated human liver after warm ischaemia: a preliminary report. Crit Care Resusc 2014;16:197-201.

84 op den Dries S, Karimian N, Sutton ME, et al: Ex vivo normothermic machine perfusion and viability testing of discarded human donor livers. Am J Transplant 2013;13:1327-1335.

85 Nassar A, Liu Q, Farias K, et al: Role of vasodilation during normothermic machine perfusion of DCD porcine livers. Int J Artif Organs 2014;37:165-172. 
86 Kelly DM, Shiba H, Nakagawa S, et al: Hepatic blood flow plays an important role in ischemia-reperfusion injury. Liver Transpl 2011;17:1448-1456.

87 Knaak JM, Spetzler VN, Selzner N, Selzner M: Normothermic machine perfusion of discarded liver grafts what is viable? Am J Transplant 2013;13:2503.

88 Izamis M-L, Tolboom H, Uygun B, et al: Resuscitation of ischemic donor livers with normothermic machine perfusion: a metabolic flux analysis of treatment in rats. PLoS One 2013;8:e69758.

89 Watson CJE, Kosmoliaptsis V, Randle LV, et al: Preimplant normothermic liver perfusion of a suboptimal liver donated after circulatory death. Am J Transplant 2016;16:353-357.

90 Ravikumar R, Jassem W, Mergental H, et al: Liver transplantation after ex vivo normothermic machine preservation: a phase 1 (first-in-man) clinical trial. Am J Transplant 2016;DOI: 10.1111/ajt.13708.
91 Vajdová K, Smreková R, Mislanová C, et al: Cold-preservation-induced sensitivity of rat hepatocyte function to rewarming injury and its prevention by short-term reperfusion. Hepatology 2000;32:289-296.

92 Reddy SP, Bhattacharjya S, Maniakin N, et al: Preservation of porcine non-heart-beating donor livers by sequential cold storage and warm perfusion. Transplantation 2004;77:1328-1332.

93 Leducq N, Delmas-Beauvieux MC, Bourdel-Marchasson I, et al: Mitochondrial permeability transition during hypothermic to normothermic reperfusion in rat liver demonstrated by the protective effect of cyclosporin A. Biochem J 1998;336:501-506.

94 Rauen U, Kerkweg U, de Groot H: Iron-dependent vs. iron-independent cold-induced injury to cultured rat hepatocytes: a comparative study in physiological media and organ preservation solutions. Cryobiology 2007; 54:77-86.
95 Shigeta T, Matsuno N, Obara H, et al: Impact of rewarming preservation by continuous machine perfusion: improved post-transplant recovery in pigs. Transplant Proc 2013;45:1684-1689.

96 Hoyer DP, Mathé Z, Gallinat A, et al: Controlled oxygenated rewarming of cold stored livers prior to transplantation: first clinical application of a new concept. Transplantation 2016;100:147-152.

97 Schlegel A, Kron P, Graf R, et al: Warm vs. cold perfusion techniques to rescue rodent liver grafts. J Hepatol 2014;61:1267-1275.

98 Matsuno N, Obara H, Watanabe R, et al: Rewarming preservation by organ perfusion system for donation after cardiac death liver grafts in pigs. Transplant Proc 2014;46:1095-1098.

99 Banan B, Xiao Z, Watson R, et al: Novel strategy to decrease reperfusion injuries and improve function of cold preserved livers using normothermic ex-vivo liver perfusion machine. Liver Transpl 2016;22:333-343. 